\title{
DAS INSTITNT DER REALOBLIGATION
}

\author{
Doç. Dr. Şapak N. EREL
}

\section{Einführung}

Die wesentliche Grundlage für den systematischen Aufbau wie für die dogmatische Durchbildung der Vermögensrechtsordnung bildet natur. gemaess das gegenseitige Verhaeltnis von Schuldrechten und Sachenrechten. Es beruht seinerseits unmittelbar auf der begrifflichen Bestimmung oder dem logischen Wesen, das diesen beiden Arten dés subjektiven Rechts eignet.

Mit der herkömmlichen Auffassung sehen wir das Kennzeichen eines dinglichen Rechts in der unmittelbaren Beherrschung oderBeherrschbar. keit eines Vermögensobjekts; nach aussen tritt sie in der Absolutheit oder in der Unabhaengigkeit des Rechts von jeder persönlichen Relation zutage. Demgegenüber erschöpft sich das Schuldrecht oder, genauer gesagt, der Anspruch als relatives Recht in einer persönlichen Beziehung zwischen dem Berechtigten und dem Verpflichteten; hier wird nicht das Vermögensobjekt selbst beherrscht, sondern allenfalls ein bestimmtes $\checkmark$ erhalten des Schuldners.

Die klare Trennung von obligatorischen und dinglichen Rechten und die Beschraenkung der Zahl dinglicher Rechtsinstitute haben bei all ihren Vorteilen den grossen Nachteil, dass sie zahlreiche Bedürfnisse des Rechtslebens nicht $\mathrm{zu}$ befriedigen vermögen. Eine systematisch - dogmatische Erklaerung muss gefunden werden für Verbindungen von Elementen des Obligationen - und Sachenrechts in dem Sinne, dass der jeweilige dinglich Berechtigte oder Besitzer als Schuldner bzw. als Glaeubiger erscheint. Auch für schuldrechtliche Verhaeltnisse ergibt sich das Bedürfnis, eine staerkere Durchsetzbarkeit, namentlich durch Realexekution, sowie gegen spaetere nachteilige Verfügungen des dinglich Berechtigten zu sichern und Rechte wie Pflichten auch gegen die Singularsukzessoren wirksam zu machen. Ohne das Prinzip von "numerus clausus" durchzubrechen, den man für die dinglichen Rechte als rotwendig erachtet, wird auch diesen Postulaten in wirksamer Weise durch das Institut der Realobligation Rechnung getragen. 
Mit den, folgenden Ausführungen wird der Versuch unternommen, die Realobligationen in ihren Verschiedenen Erscheinungsformen als Rechtsinstitut von ausgepraegter Eigenart darzustellen, dessen Wurzeln weit in die Rechtsgeschichtliche Vergarngenheit zurückreichen. So reizvoll es sein mag, dies in der umfassenden Anwendung des Institutes zu tun, so liegt es nahe, uns hierbei auf das Gebiet des Sachenrechts zu beschraenken, da eine einheitliche Betrachtung und Zusammenfassung überhaupt nur in dieser Beschraenkung Aussicht auf Erfolg bietet.

\section{Die Rechtsnatur der Realobligation}

Die schweizerische und türkische Zivilgesetzbücher sehen zahlreiche Faelle vor, in denen dem jeweiliger Inhaber eines dinglichen Rechts unmittelbar kraft Gesetzes oder aur. Grund eines Rechtsgeschaeftes Leistungspflichten auferlegt werden. Aber für diese Situationen fehlt eine gesetzliche Terminologie. Da dem Gesetzgeber die gemeinsame systematisch -dogmatische Grundlage dieser Obligationen nicht bekannt war, erfasste er sie nicht als eine besondere Kategorie. Zur Kennzeichnung der subjektiv-dinglichen Verbindung nennt man diese Leistungspflichten in Deutschland und deutsch-spraechige Schweiz "Realobligation" und in Frankreich und fransözisch-spraechige Schweiz "Obligation propter rem".

Die Realobligation ist kein dingliches Recht. Die absolute Wirkung der dinglichen Recht gibt dem Berechtigten die Macht jedermann auszuschliessen. Aber wenn ein Recht eine bestimmte Wirkung nur zwischen den Parteien entfaltet, ist es ein relàtives Recht. Die Leistungspflicht ist Gegenstand eines relatives Rechtsverhaeltnisses. Bei der Realobligation ist nur der Parteibegriff auf der Passivseite erweitert, da nicht eine bestimmte Person, sondern der jeweilige Inhaber eines dinglichen Rechts Schuldner wird. Dies aber aendert an der relativen Natur des Rechtsverhaeltnisses nichts. Die Realobligaiton wirkt nur gegen denjenigen, der durch Erwerb des dinglichen Rechts in das relative Schuldverhaeltnis eintritt. ${ }^{1}$ Wo immer die Sachherrschaft darin besteht, dass eine bestimmte Person zu einem Tun oder Handeln verpflichtet ist. liegt kein dingliches Recht vor. Die Realobligaiton verschafft keine Sachherrschaft und ihr entspricht auch keine Einschraenkung der Sachherrschaft der Verpflichteten. Nicht seine Sachherrschaft, sondern seine persönliche Handlungsfreiheit wird betroffen. ${ }^{2}$ Die Realobligaiton

1 Liver: ZBGR 43, s. 262; Haab : Einl. N. 54; Ostertag: Art. 959, N. 10; Homberger: Art. 859, N. 3; Guhl : Festgabe, s. 121 vd.

2 Liver: ZBGR 43, s. 262 vd., Komm. Einl. N. 148, 153, Art. 730, N. 225; Liver: ZBJV 98, s. 212, 227; Jost: s. 2 vd., 60; Meier-Hayoz: Syst. Teil N. 150; Haab: Einl. N. 54; Neuenschwander: s. 108 vd., 111 vd. 
bildet eine Beziehung zwischen zwei Personen, kraft welcher die eine Seite zu einem bestimmten Verhalten. verpflichtet ist. Sie wirkt nur gegen den jeweils Verpflichteten. Der jeweils Verpflichtete haftet für die Erfüllung der Schuld grundsaetzlich persönlich und unbeschraenkt. Wenn der jeweilige dinglich Berechtigte zu einem Dulden oder Unterlassen verpflichtet ist, so ist seine Sachherrschaft beschraenkt; dieser Fall entspricht nicht eine Realobligaton, sondern ein beschraenktes dingliches Recht oder eine Eigentumsbeschraenkung. ${ }^{3}$

Auch der Inhaber des dinglichen Rechts hat zur Durchsetzung seines Rechts dingliche Ansprüche, aber diese gehen auf Herausgabe der Sache oder auf Beseitigung und Unterlassung von Beeintraechtigungen. Diese Ansprüche richten sich nicht gegen den jeweiliger dinglich Berechtigten, sondern gegen den jeweiligen Störer. Der Störer liegt nicht unter einer realobligatorischen Leistungspflicht, sondern zur Beachtung des dinglichen Rechts. ${ }^{4}$ Eine unerlaubte Handlung oder Haftung zum Ersatz des Schadens kann nicht Inhalt oder Gegenstand der Realobligation sein.

Die aus der relativrechtlichen Natur der Realobligation entstehende Rechtsfolgen sind, dass der Schuldner durch eine dingliche Berechtigung bestimmt wird, dass die Schuldpflicht deshalb zusammen mit dem dingiichen Recht übertragen wird, und die Haftung aufhört sobald das dingliche Recht durch Übertragung oder Verzicht untergegangen ist, und schliesslich dass die Realobligation infolge ihrer engen Verknüpfung mit dinglichen Rechten an der Typengeburdenheit der Sachenrechte teil hat. in der Doktrin wurde wenigstens eingesehen, dass durch die Vormerkung persönlicher Rechte - ein typischer Fall der Realobligation- deren obligatorische Rechtsnatur nicht veraendert wird.

\section{Die Glaeubiger und Schuldnerstellung bei der Realobligation}

Das Gesetz kann die Glaeubiger oder Schuldnereigenschaft mit einer dinglichen Berechtigung verbinden, aber da der Charakter einer obligation sich einzig nach der speziellen Bezeichnung der Schuldnerschaft bestimmt und die Bezeichnung des Glaeubigers ohne Einfluss ist, ist die

3 Die alte Doktrin nennt auch Leistungspfiichten als Inhalt von Eigentumsbeschraenkungen: Wieland: Art. 680 Bem. 3; Leemann: Art. 680, N. 19, 23, Haab: Art. 641, N. 3, 11; Art. 680, N. 26; siehe Meier-Hayoz: Art. 641, N. 33.

- von Tuhr: Bd. I \&2, IV 2b, s. 12 vd., Wieland: Vorbem. zum IV Teil, s. 1; Haab: Einl. N. 55; Liver : ZBJV 98, s. 227; Deschenaux: Festgabe, s. 717; Jost: s. 56.

5 Guhl : Festgabe, s. 130, 164 vd.; Haab: Eirl., 54, Art. 681, N. 8; Leemann: Einl. I, N. 26, Art. 681, N. 6, Vorbem. 782ff. N. 4; Homberger: Art. 958, N. 3, 17; Wieland: Art. 681, Bem. 2, Art. 959, Bem. 1. 
Glaeubigerstellung in einem realobligatorischen Rechtsverhaeltnis nicht Wesensmerkmal. Wenn das Gesetz den jeweiligen Inhaber eines dinglichen Rechts als Glaeubiger bezeichnet, so findet mit der Übertragung des dinglichen Rechts ein gesetzlicher Forderungsübergang statt. Der Erfüllungsanspruch des Dritten, der in die Stellung eintritt, gehorcht den Regeln der Zession oder des Vertrages zugunsten Dritter. Abreden die den jeweiligen dinglich Berechtigten als Glaeubiger bezeichnen bedürfen keiner gesetzlichen Grundlage, weil die Bezeichnung der Glaeubigerschaft ein aeusserer Umstand ist, und dadurch das Wesen der Obligation nicht veraendert wird. Immerhin wird in der Doktrin im allgemeinen von einer Realobligation gesprochen, wenn der Shuldner oder der Glaeubiger oder beide durch eine dingliche Berechtigung bestimmt werden. ${ }^{6}$

Die Realobligation hat grundsaetzlich den Grundeigentümer zum Schuldner. Aber auch der Inhaber voin beschraenkten dinglichen Rechte oder der Besitzer kann die Schuldnerstellung erwerben. Der Miteigentümer schuldet zusammen mit seinen Partnern die Leistungen des Eigentümers, die besonders aus Nachbarrecht entstehen. Leistungspflichten des Besitzers wird in der Doktrin im. allgemeinen bejaht. ${ }^{7}$ Auf Grund des Besitzes wird eine subjektiv-dingliche Leistung geschuldet, wenn das Gesetz direkt den Besitzer zu einer Leistung verpflichtet. Dies geschiet im Falle des Eigenbesitzes, z.B. wenn das Gesetz dem Besitzer einer verlorenen und zugeführten Sache positive Pflichten auferlegt (ZGB 720, 725, MK 693, 698). Der Eigen Besitze: hat die Gewalt über eine Sache und den Willen, sie als Eigentümer oder dinglich beschraenkt Berechtigter $z u$ besitzen, ohne der echte Eigentümer cder Berechtigter zu sein brauchen. Je nach seinem Willen, schuldet ęr die Leistungen eines Eigentümers oder dinglich beschraenkt Berechtigten und er kann sich seiner Haftung nicht entschlagen mit der Behauptung, dass er noch kein dingliches Recht ersessen hat. ${ }^{8}$ Leistungspflichten des dinglich beschraenkt Beréchtigten richten sich regelmaesig auf der Werterhaltung der belasteten Sache, und er schuldet dem Eigentümer des Grundstückes.

Für den Beweis der Passiv sciwohl Aktivlegitimation bei der Glaeubiger bzw. Schuldnerstellung genügt der Nachweis der dinglichen Berechtigung. Wenn die Leistungspflicht mit dem dinglichen Recht an einem Grundstück verbunden ist, muss der Glaeubiger den Nachweis erbringen, dass der Schuldner im Grundbuch eingetragen ist; oder dass

${ }^{6}$ Meier-Hayoz: Syst. Teil, N. 152, 159; Liver: Einl. N. 148, ZBGR 43, s. 261; Guhl: Festgabe, s. 119 vd.; Jost: s. 45, 95; Neuenschwander: s. 118 vd.

${ }^{7}$ Liver: Einl. N. 148, 156, 163, ZBGR 43, 5. 261; Meier-Hayoz: Syst. Teil, 152 f. 159, f.; Deschenaux: Festgabe, s. 726.

\$ Jost : s. 125 f; Deschenaux: Festgabe, s. 726; Neuenschwainder: s. 122, 123. 
er das dingliche Recht aussergrundbuchlich erworben hat (ZGB 937/I, 656/I, MK 905, 633/I). Für das Fahrnisrecht kann der Glaeubiger sich auf die Vermutung aus dem Besitz berufen (ZGB 930, ff, MK 898 ff). $?$ Der an einem Grundstück obligatorisch berechtigte (Mieter, Paechter usw.) kann der Glaeubiger, aber niemals der Schuldner einer realobligatorischen Leistung sein.

\section{Die Übertragung der Leistungspflicht}

Die Schuld entsteht in der Person des Singularsukzessors ohne Willenserklaerung einer der beteiligten. Utberhaupt keine Willensbildung in Bezug auf den Schuldübergang ist nötig, und meistens nur der Inhaber der Sachherrschaft wird in der Lage sein, die geschuldete Leistung zu erbringen. Der bisherige Schuldner befreit sich durch die Veraeusserung seines dinglichen Rechts von den noch nicht faellig gewordenen Schulden.

Für den Eintritt des Erwerbers des dinglichen Rechts in die damit verbundene Schuldpflicht, ist der Rechtsgrund des Erwerbs ohne Bedeutung. Sobald der Erwerber die Voraussetzungen erfüllt, an die das Gesetz oder der Vertrag die Schuldrerstellung knüpfen, so ist er der Schuldner aus der Realobligation, abgesehen davon ob er das dingliche Recht originaer oder derivativ erworken hat.

Die Entstehung und Übertragung der gesetzlichen Realobligationen setzt keine grundbuchliche Publizitact voraus. Unmittelbar durch das Gesetz begründete Leistungspflichten sind weder eintraguns-noch vormerkungsfaehig. Ein Bedürfnis nach einer grundbuchlichen Publizitaet besteht nicht, weil mit dem Erwerb des dinglichen Rechts auch die mit diesem gesetzlich verbundene Verpflichtung ensteht und jeder Erwerber damit rechnen muss. ${ }^{10}$ Die realobligatorische Wirkung von vertraglichen Leistungpflichten ist dagegen von einer Publikation abhaengig. Wenn das Gesetz für den Übergang der Leistungspflicht auf den Erwerber des dinglichen Rechts eine Publikation verlangt, ist die Publikation für die Wirkung gegenüber dem Erwerber konstitutiv. Ohne Publikation würde die Schuld an der Person haften bleiken und erst die Publikation stellt eine Verbindung zwischen Schuld und dinglichem Recht her. Der Erwerber hat hier Gewissheit, dass nicht publizierte Leistungspflichten für ihn unverbindlich sind.

In der Regel wird der bisherige Schuldner nach Treu und Glauben verpflichtet sein, dem Glaeubiger die Handaenderung zu notifizieren. Diese Pflicht ist Bestandteil des realobligatorischen Schuldverhaeltnisses.

9 Jost: s. 117, 125; Haab: Art. 679, N. 11; Wieland: Art. 745, Bem. 3.

${ }^{10}$ Liver: ZBGR 43, s. 275; Botschaft des Eundesrates vom 9.4.1963 über den Revisionsentwurf betreffend das Baurecht, S. 13. 
Wenn der Glaeubiger infolge Missachtung der Mitteilungspflicht Schaden erleidet, wird der bisherige Schuldner ersatzpflichtig. Beim Vorkaufsrecht ist die Mitteilungspflicht vom Gesetz ausdrücklich geregelt (ZGB 681/II, MK 658/II). Der Glaeubiger kann auf den Übergang der Schuld keinen Einfluss ausüben. En hat dem Schuldubergang weder zuzustimmen, noch kann er ihn durch seinen Einspruch verhindern. ${ }^{11}$

Grundsaetzlich werden Mieter und Paechter, Nutzniesser und andere dritte Personen, die in eine rechtliche Beziehung zu einem Grundstück treten, dessen jeweiliger Eigentümer schüldet, nicht Schuldner aus diesen Realobligationen. Wenn sie die Leistungen erbringen, handeln sie als Erfüllungsvertretern. ${ }^{12}$ Für die realobligatorischen Leistungspflichten des Eigentümers bewırkt der Miet-und Pachtvertrag eine interne Schuldübernahme, und im externen Verhaeltnis bleibt der Eigentümer Schuldner aus den Realobligationen.

Die Erklaerung des Schuldübergangs ist aus dem Begriff der Realobligation abzuleiten. Da der jeweilige Inhaber des dinglichen Rechts Schuldner aus der Realobligation ist, tritt mit der Handaenderung des dinglichen Rechts anstelle des bisher!gen Inhaber der Erwerber in die Stellung des Schuldners ein. Aber am Schuldverhaeltnis aendert sich nichts. Der frühere Schuldner hat die Stellung verloren, an die das Gesetz oder der Vertrag die Schuldnerschaft knüpfen, der Erwerber ist in die Stellung eingetreten und ist dadurch zum Schuldner geworden. Dies ist nicht eine Novation mit Schuldnerwechsel, wie Meier-Hayoz und Deschenaux meinen, sondern eine Rechtsnachfolge mit fortbestehendem Schuldverhaeltnis. ${ }^{13}$ Der Schuldübergang ist privativ, d.h. der Erwerber tritt nicht neben den Veraeuserer, sondern nimmt seine Position ein. Schadenersatzpflichten aus Nicht- lind Schlechterfüllung, sowie die faellige Leistungspflichten bilden persönliche Forderungen und verbleiben dem bisherigen Schuldner, auch wenn dieser sein dingliches Recht veaeussert. ${ }^{14}$

\section{Das Prinzip von Numerus Clausus bei den Realobligationen}

Die Doktrin fordert die geschlossene Zahl und die Typengebundenheit der Realobligationen..$^{15}$ Das bedeutet der Ausschluss der Vertragsfreiheit

${ }_{11}$ Liver: Art. 730, N. 232; Meier-Hayoz: Syste. Teil, N. 161.

12 Liver: Art. 730, N. 228; Jost: S. 119, 127.

13 Meier-Hayoz: Syst. Teil, N. 161, 177; Deschenaux : Festgabe. S. 741.

14 Liver: Einl. N. 163; Jost: S. 103; Meier-Hayoz: Syst. Teil, N. 162, Art. 649, N. 9; Deschenaux : Festgabe, S. 742-744.

15 Liver: Einl. N. 150f, Art. 730, N. 157, ZBGR 43, S. 277; Meier-Hayoz: Syst. Teil, N. 153, 165f: Jost: S. 65, 97ff; Deschenaux: Festgabe, S. 717f, ZBGR 43, S. 285. 
für die Begründung realobligatorischer Leistungspflichten. Aber die Realobligationen unterstehen nicht dem sachenrechtlichen numerus clausus, sondern der numerus clausus der Realobligationen findet lediglich seine Erklaerung und Rechtfertigung im sachenrechtlichen numerus clausus. Realobligationen erfordern, wegen der verstaerkten Wirkung gegen den Dritten, eine gewisse Publizitaet. Der Ausschluss der Vertragsfreiheit kann also mit aehnlichen Erwaegunge $\mathbf{n}$. begründet werden wie für die dinglichen Rechts. Das Rechtsgeschaef $t$ vermag nur dann eine Realobligation zu begründen, wenn das Gesetz dies erlaubt. Auch in der Inhaltlichen Ausgestaltung der Realobligation, der numerus clausus bindet die Parteien; ausserhalb der gesetzlichen Typen können sie den Leistungspflichten keine realobligatorische Wirkung beilegen. Die Parteien müssen sich an die vom Gesetz zur Verrügung gestellten und definierten Typen rechtsgeschaeftlicher Realobligationen halten. Dieses Prinzip ist auch positivrechtlich festgelegt: Gemaess Art. 959 ZGB (Art. 919 MK) können cbligatorische Rechte durch Vormerkung mit einem dinglichen Recht verbunden werden, "wenn die Vormerkung durch das Gesetz ausdrücklich vorgesehen ist."

\section{Inhalt der Realobligationen}

Inhalt einer Realobligation kann nur positive Leistungspflichten sein, nicht aber Unterlassungs oder Duldungspflichten. Linterlassungs - und Duldungspflichten des jeweiligen dinglich Berechtigten schraenken seine Sachherrschaft ein und bilden daher eine Dienstbarkeit oder eine Eigentumsbeschraenkung. Die positive Leistungspflicht als Inhalt der Realobligation ist ein Begriffsmerkmäi, denn sie bildet das Kriterium ihrer Unterscheidung von den Dienstbarkeiten und Eigentumsbeschraenkungen. ${ }^{16}$ Worin besteht dann dise positive Leistungspflichten?

Inhalt der gesétzlichen Realobligationen können Arbeitsleistungen, Geldleistungen oder die Leistungspflichten zur Abgabe einer rechtsgeschaeflichen Willenserklaerung sein. Arbeitsleistungen die nicht vom Schuldner persönlich erbracht werden müssen bilden haeufiger den Inhalt einer Realobligation; z.B. Unterhaltspflichten des Nutzniessers, des Faustpfandglaeubigers, Grenzmarkierung, Bau und Unterhalt von Grenzmauern, Mitwirkungspflichten der Mit - und Stockwerkeigentümern bei der Organization und Verwalturg der gemeinsamen Sache. Auch Geldleistungen können Inhalt gesetzlicher Realobligationen sein; z.B. die

295; für die vorgemerkten persönlichen Rechten Homberger: Art. 959, N. 4f; Wieland: Art. 959, Bem. 2.

16 Meier-Hayoz : Syst. Teil, N. 152; Jost : S. 95; Liver : ZBGR 43, S. 263; Deschenaux: ZBGR 43, S. 282. 
Entschaedigung für die Einraeumung eines dinglichen Rechts auf Grund gesetzlichen Zwangs (Notdienstbarkeiten), der Anteil an den Abmarkungskosten, der Kostenbeitrag der Mit- und Stockwerkeigentümer. Noch wichtiger sind die Leistungspflichten zur Abgabe einer rechtsgeschaeftlichen Willenserklaerung: das Gesetz verpflichtet den Grundeigentümer zur Einraeumung eines beschraenkten dinglichen Rechts oder zur Abtretung von Eigentum an seinem Grundstück, wie z.B. die Anmeldung zur Eintragung von Notdiensbarkeiten und mittelbar gesetzlichen Grundpfandrechten oder gemaess dem Vorkaufsrecnt, Eintragugsanmeldung zugursten des Vorkaufsberechtigten.

Bei rechtsgeschaeflichen Realobligationen sind die Parteien frei, den Inhalt der Leistungspflichten auszugestalten. Aus dem numerus clausus ergeben sich aber Schranken. Das Vertrauensprinzip ist auch für die Auslegung von rechtsgeschaeflichen healobligationen grundlegend, weil der gutglaeubige neu Schuldner geschützt werden muss. Massgebend ist die Erklaerung in Grundbuchbelegen und wie sie der gegenwaertige Schuldner nach Treu und Glauben verstehen musste. Verwaltungsreglemente unter Mit- und Stockwerkeigentümer sind wie Gesellschaftsstatuten auszulegen. Auch Mehrheitsbeschlüsse, die eine Leistungspflicht begründen, sind wie Gesellschaftsbeschlüsse auszulegen. ${ }^{17}$

\section{Rang der Realobligationen}

Nach schweizerischem und türkischem Recht erhalten die dingliche Rechte nach dem Datum des Grundbucheintrags einen Rang (ZGB 972, MK 930); mit der Ausnahme von Grundpfandrechte unter sich. Deren Rang wird nach den Pfandstellen besiimmt (ZGB 813ff, MK 785ff.). In Doktrin wird die Ansicht vertreten, ciass den Realobligationen kommen unter sich und im Verhaeltnis zu dinglichen Rechten ein Rang zu. Realobligationen unterstehen dem Prinzip der Aeltersprioriataet im Falle einer Konkurrenz sowohl zwischen mehreren realobligatorischen Ansprüchen, als auch zwischen einem dinglichen Recht und einem realobligatorischen Anspruch gleich wie bei den dinglichen Rechten unter sich. ${ }^{18}$

Wir müssen demgegenüber ablehnen, von einer Rangordnung als Merkmal der Realobligation zu sprechen. Die Realobligation ist ein. obligatorisches Recht; sie ist gegen di Person des Schuldners und nicht unmittelbar auf eine Sache gerichtet. Erst im Vollstreckungsverfahren ist eine Kollision zwischen dinglichen Rechten und Realobligationen

\footnotetext{
"Meier-Hayoz: Syst. Teil, N. 170-171; Neuenschwander: S. 134, 230-231.

18 Meier-Hayoz: Syst. Teil, N. 153, 167-168; Jost: S. 100, 101f; bei Vormerkungen: Jost : S. 156f, 168; Ostertag : Art. 959, N. $8 f$.
} 
möglich, da die Leistungspflichten dəs Schuldners sowie seine Rechte als Bestandteiie seines Vermögens den Glaeubigern haften. Diese Kollision tritt aber auch zwischen Ansprüchen aus persönlichen Obligationen und dinglichen Rechten ein. Nur von der'Einraeumung einer gesetzlichen Dienstbarkeit werden die Inhaber beschraenkter dinglicher Rechte betroffen, doch, hier habe die auf Grund d $\epsilon$ s gesetzlichen Anspruchs errichtete Dienstbarkeit und nicht der Anspruch einen Rang. Auch im falle der vorgemerkten obligatorischen Rechte komme der Rang nicht der Realobligation, sondern dem vorgemerkten Recht zu. Spaeter begründete dingliche Rechte, wrelche die Erfüllung der Leistungspflicht durch den ursprünglichen Schuldner nicht ausschliessen, aber den Anspruch beeintraechtigen, sind dem vormerkungsberechtigten Glaeubiger gegenüber insoweit nicht wirksam. Das vergemerkte persönliche Recht hat einen Rang infolge der durch die Vormerkung bewirkten Verfügunsbeschraenkung. Die aus dem vorgemerkten Recht entstehenden realobligatorischen Leistungspflichten nehmen dagegen an der Rangordnung der dinglichen Rechte nicht teil. ${ }^{19}$

8. Die Wirkungen des Pfaendungs- und Konkursbeschlages Gegenüber Realobligationen

Wie oben dargelegt wurde, bestimmte Realobligationen verpflichten den jeweiligen Eigentümer eines Grundstückes, dem Berechtigten ein dingliches Recht am Grundstück einzuraeumen. Dazu gehören u.a. die sogenannten mittelbaren Eigentumsbeschraenkungen, naemlich die Verpflichtung zur Einraeumung des Überbaurechts oder zur Abtretung des überbauten Bodens (ZGB 674/III, MK 651/II), sowie die Gewaehrung des Durchleitungsrechts (ZGB 710, MK 683); ferner bestimmte im Grundbuch vorgemerkte persönliche Rechte wie das Kaufsrecht, Vor- und Rückkaufsrecht (ZGB 683, MK 660) oder das Rückfallrecht bei der Schenkung (OR 247/II, BK 242/II).

Fraglich ist, welche Wirkungen die Pfaendung und Admassierung einer Sache haben, deren Eigentümer auf Grund einer solchen Realobligation zur Einraeumung eines dinglichen Rechts verpflichtet ist. Die erwaehnten Realobligationen belasten naemlich das betreffende Grundstück wie ein dingliches Recht, indem der Eigentümer das Grundstück nicht veraeussern kann, ohne dass sie als "Last" auf den Erwerber übergehen. Dies trifft auch zu, wenn das Grundstück im Pfaendungs- oder Konkursverfahren zwangsweise verwertet wird. Es waere daher sinnlos,

${ }_{19}$ Deschenaux : Festgabe, S. 719ff, ZBGR 43, S. 284f; Neuenschwander: S. 134-137. 
den Anspruch aus einer solehen Realobligation bei der Pfaendung und im Konkurs wie einen gewöhnlichen Anspruch auf Begründung eines dinglichen Rechts zu behandeln; denn nichts hinderte den aus der Realobligation Berechtigten, seinen Anspruch auch gegen den, der die Liegenschaft aus der Pfaendungs- oder Konirurmasse erworben hat, geltend zu machen und von ihm den Eintrag zu verlangen. Aus diesem Grund halten die genannten Realobligationen auf Begründung eines dinglichen Rechts gegenüber den spaeter entstandenen Beschlagsrechten der Glaeubiger stand, sogar die Ansprüche auf Errichtung einer Notdienstbarkeit gehen den früher entstandenen Beschlagsrechte vor. ${ }^{20}$

In dieser Hinsicht gibt es zwei Unterschiede zwischen den Persönlichen - und Realobligationen: der aus der Realobligation Berechtigte kann waehrend des Pfaendungs- und Konkursverfahrens, ohne Rücksicht auf die Beschlagsrechte der Glaeisbiger die Begründung des dinglichen Rechts verlangen, und er kann seinen Anspruch auf die Begründung des dinglichen Rechts gegen den neuen Eigentümer, der lie Liegenschaft aus der Pfaendungs- oder Konkurme.sse erworben hat, geltend machen.

Der Anspruch auf Erric.itung einer Legalservitut (Notdienstbarkeit). wird zum Eintrag ins Lastenverzeichnis angemeldet, und zwar als Last, die allen übrigen Belastungen vorgeht. Das nachfolgende Lastenbereinigungsverfahren gibt den betroffenen Inhabern von beschraenkten dinglichen Rechten und den Pfaendungsglaebigern Gelegenheit, Bestand und Rang der angemeldeten Servitut zu bestreiten. (SchKG Art. 140/II, IIKK md. 128) ${ }^{21}$ Dabei steht ihnerı auch der Einwand offen, der Anspruch auf die Errichtung der Legalservitut habe gar nich bestanden. Da sie die Realobligation im Gegensatz $z u$ den anderen Lasten nicht bestreiten können, muss ihnen mindestens dafür die Möglichkeit belassen werden, die Realobligation als Voraussetzung der Legalservitut $\mathrm{zu}$ bestreiten. Damit wird die Wegweisung der Legalservitut aus dem Lastenverzeichnis erreicht. Wird dagegen diese im Lastenverzeichnis anerkannt, so faellt die dem Eigentümer zusteinende Entschaedigungsforderung ipso iure unter die Pfaendung, ohne dass es eine Nachpfaendung bedarf. Das Betreibungsamt wird bei der Verwertung des Grundstückes, die im_Lastenverzeichnis rechtskraeftig anerkannte Servitut zur Eintragung im Grundbuch anmelden. Bei dem Konkursverfahren, ist die Legalservitut. von der Konkursverwaltung in das Lastenverzeichnis, das im Konkursverfahren

${ }^{20}$ Für die vorgemerkten persönlichen Rechten ist dies allgemein anerkannt: Jost: S. 157; Homberger: Art. 959, N. 30; Haab: Art. 683, N. 9ff; Guhl: Festgabe. S. 165.

${ }^{21}$ Siehe Jast: S. 135, 146; Liver: Art. 730, N. 94f, 98f. 
€inen Bestandteil des Kollokationsplanes bildet, aufzunehmen und dann aehnlich wie beim Pfaendungsverfahren, von den beschraenkt dinglich Berechtigten und Konkursglaeubigern angefochten werden. Dabei faellt die dem Eigentümer zustehende Entschaedigung ohne weiteres in die Masse.

Der Anspruch auf Errichtung eines gesetzlichen Grundpfandrechts geht stets dem Pfaendungs- und Konkursbeschlag vor, selbst wenn er erst nach der Pfaendung oder der Konkurseröffnung entstanden ist. Der Schuldner kann das Grundstück nur mit der darauf lastenden Realobligation, die den Erwerber zum Eintrag des Grundpfandrechtes verpflichtet, veraeussern. Diese Sachlage müssen sich auch die Pfaendungs- und Konkursglaeubiger entgegenhalten lassen, wenn das Grundstück zu ihrer Befriedigung zwangsweise veraeussert wird. ${ }^{22}$ Die Eintragung der mittelbar gesetzlichen Grundpfandrechte bedarf der Zustimmung des Grundeigentümers. Dies gilt auch für den Eintrag nach erfolgter Pfaendung des Grundstückes. Hingegen hat im Konkursverfahren nicht der Eigentümer, sondern an seiner Stelle die Konkursverwaltung ihre Zustimmung. zum Eintrag zu erteilen.

Im Gegensatz $\mathrm{zu}$ den gesetzlichen Realobligationen, werden Kaufsund Rückkaufsrecht als rechtsgeschaeftliche Realobligation im Grundbuch vorgemerkt und auch in das Lastenverzeichnis aufgenommen. Der Berechtigte kann sein vorgemerktes Kaufs-oder Rückkaufsrecht (nicht aber sein Vorkaufsrecht), nicht nur gegenüber dem Erwerber des Grundstückes nach durchgeführter Zwangsverwertung, sondern auch waehrend des Pfaendungs- und Konkursverfahrens ausüben. ${ }^{23}$ Die Inhaber von beschraenkten dinglichen Rechten und die Pfaendungsglaeubiger haben die Gelegenheit, das behauptete Recht im Lastenverzeichnis zu bestreiten. Bis zur rechtskraeftigen Anerkennung des Kaufrechts im Lastenverzeichnis, bleibt das Grundstück ohne Rücksicht auf den Eigentümerwechsel gepfaendet und wird nach erfolgreicher Bestreitung des Kaufrechts verwertet. Nach der rechtskraeftiger Anerkennung des Kaufrechts faellt der vom Kaufsberechtigten zu entrichtende Kaufpreis in die Pfaendungsmasse, bzw. in die Konkursmasse.

$\overline{22}$ Liver: ZBJV 98 (1962), S. 233; Staehelin. S. 74f.; a.M. Guhl : Festgabe, S. 157f.; Deschenaux: Festgabe, S. 752.

23 BGE 44 II 371ff.; Ostertag: Art. 959, N. 7; Guhl: Festgabe, S. 165, 172; Haab : Art. 683, N. 8; Homberger: Art. 958, N. 20, Staehelin: S. 76-78. 


\section{LITERATURVERZEICHNIS}

DESCHENAUX, Henri

DULCKEIT, Gerhard

GUHL, Theo

HA.AB, Robert

HOMBERGER, A.

HUBER, Eugen

JOST, Arthur

JUGLART, de Michel

LEEMANN, Hans

LENT

LIVER, Peter

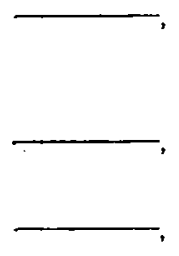

MEIER-HAYOZ, Arthur
Obligations Propter Rem (Ius et Lex), Festgabe für Max Gutzwiller, Basel 1959, S. $711 \mathrm{ff}$.

: Les obligations dites réelles, ZBGR 43 (1962), S. $282 \mathrm{ff}$.

: Die Vendinglichung obligatorischer Rechte, Tübingen 1951.

: Persönliche Rechte mit verstaerkter Wirkung (Festgabe der jur. Fakultaet für das schweiz. Bundesgericht S. 93-178). Bern 1924.

: Kommentar zum ZGB, IV Band Das Sachenrecht, Zürich 1929.

: Kommentar zum ZGB, IV Band Das Sachenrecht, II. Abteilung Besitz und Grundbuch, Bern 1938.

: Zum schweizerischen Sachenrecht Drei Vortraege mit Anmerkungen, Bern 1914.

: Die Realobligation als Rechtsintitut, Bern 1956.

: Obligations réelle et servitudes en droit privé français, These Bordeaux 1937.

: Kommentar zum schweiz. ZGB, IV Band Sachenrecht, Erste Abteilung Bern 1920, Zweite Abteilung Bern 1925.

: Zwangsvollstreckungs- und Konkursrecht, 7. Auflage München-Berlin 1958.

: Gesetzliche Eigentumsbeschraenkungen und Dienstbarkeiten in der Gesetzgebung und Lehre Frankreichs, Deutschlands, der Schweiz und Italiens, (Ius et Lex), Festgabe für Max Gutzwiller, Basel 1959 , S. $749 \mathrm{ff}$.

: Kommentar zum schweiz. ZGB, IV Band Das Sachenrecht, Die Dienstbarkeiten und Grundlasten, 2. Auflage Zürich 1968.

: Die Begründung des Bauhandwerkerpfandrechts, ZBJV 98 (1962). S. $20 \dot{\mathrm{ff}}$.

Die Realobligation, Ihre klassifikatorische Bedeutung und praktische Verwendbarkeit, ZBGR 43 (1962), S. 257-281.

Berner Kommenter, Band IV Sachenrecht I. Abteilung Das Eigentum, 1. Teilband Systematischer Teil, Bern 1966, 2. Teilband Das Grundeigentum, I. Bern 1965, 3. Teil band Das Grundeigentum II, Bern 1975.

NEUENSCHWANDER, U. : Die Leistungspflichten der Grundeigentümer im fransözischen CC. und im schweiz. ZGB, Bern/Zürich 1966. 
OSTERTAG, Fritz

PIOTET, Paul

SCHACCHI, Diego

STAEHELIN, Adrian

EREL, Şafak

OĞUZMAN, Kemal
: Kommentar zum schweiz. ZGB, Band IV Sachenrecht, 3. Abteilung: Besitz und Grundbuch, 2. Auflage Bern 1917.

: Les effets typiques des annotations au registre foncier, ZBGR 50, S. 34ff.

: L'obligation propter rem et les droits personnels annotes au registre foncier, these, Locarno 1970.

: Probleme aus dem Grenzbereich zwischen Privatund Zwangsvollstreckungsrecht, Basel/Stuttgart 1968.

: Eşyaỵa Bağlı Borç, Ankara 1982.

: Eşyaya Bağlı Borçlara Hakim Olan Esaslar, 1.Ü. Mukayeseli Hukuk Enstitüsü Medeni Kanun 50. Yıl Sempozyumu, Ist. 1978. 